Article

\title{
Surgery for Coagulopathy-Related Intracerebral Hemorrhage: Craniotomy vs. Minimally Invasive Neurosurgery
}

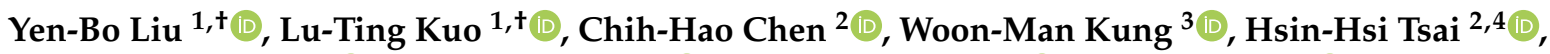 \\ Sheng-Chieh Chou ${ }^{5} \mathbb{D}$, Shih-Hung Yang ${ }^{1}{ }^{(\mathbb{D}}$, Kuo-Chuan Wang ${ }^{1} \mathbb{D}$, Dar-Ming Lai ${ }^{1} \mathbb{D}$ and Abel Po-Hao Huang ${ }^{1, *}$
}

Citation: Liu, Y.-B.; Kuo, L.-T.; Chen, C.-H.; Kung, W.-M.; Tsai, H.-H.; Chou, S.-C.; Yang, S.-H.; Wang, K.-C.; Lai, D.-M.; Huang, A.P.-H. Surgery for Coagulopathy-Related Intracerebral Hemorrhage: Craniotomy vs. Minimally Invasive Neurosurgery. Life 2021, 11, 564. https://doi.org/10.3390/ life11060564

Academic Editors: Pedro Castro and Elsa Azevedo

Received: 11 May 2021

Accepted: 9 June 2021

Published: 15 June 2021

Publisher's Note: MDPI stays neutral with regard to jurisdictional claims in published maps and institutional affiliations.

Copyright: (c) 2021 by the authors. Licensee MDPI, Basel, Switzerland. This article is an open access article distributed under the terms and conditions of the Creative Commons Attribution (CC BY) license (https:/ / creativecommons.org/licenses/by/ $4.0 /)$.
1 Division of Neurosurgery, Department of Surgery, National Taiwan University Hospital, College of Medicine, National Taiwan University, Taipei 10617, Taiwan; imountainpig@ntuh.gov.tw (Y.-B.L.); ltkuo@ntuh.gov.tw (L.-T.K.); swonyang@ntuh.gov.tw (S.-H.Y.); wang081466@ntuh.gov.tw (K.-C.W.); laidd@ntuh.gov.tw (D.-M.L.)

2 Department of Neurology, National Taiwan University Hospital, College of Medicine, National Taiwan University, Taipei 10617, Taiwan; moulinrouge@ntuh.gov.tw (C.-H.C.); hsinhsi@ntuh.gov.tw (H.-H.T.)

3 Department of Exercise and Health Promotion, College of Kinesiology and Health, Chinese Culture University, Taipei 11114, Taiwan; nskungwm@yahoo.com.tw

4 Department of Neurology, National Taiwan University Hospital Bei-Hu Branch, Taipei 10617, Taiwan

5 Division of Hematology, Department of Internal Medicine, National Taiwan University Hospital, College of Medicine, National Taiwan University, Taipei 10617, Taiwan; choushengchieh@ntuh.gov.tw

* Correspondence: how0622@ntuh.gov.tw; Tel.: +886-928-778-778

+ These authors contributed equally to this work.

Abstract: Coagulopathy-related intracerebral hemorrhage (ICH) is life-threatening. Recent studies have shown promising results with minimally invasive neurosurgery (MIN) in the reduction of mortality and improvement of functional outcomes, but no published data have recorded the safety and efficacy of MIN for coagulopathy-related ICH. Seventy-five coagulopathy-related ICH patients were retrospectively reviewed to compare the surgical outcomes between craniotomy $(n=52)$ and MIN ( $n=23)$. Postoperative rebleeding rates, morbidity rates, and mortality at 1 month were analyzed. Postoperative Glasgow Outcome Scale Extended (GOSE) and modified Rankin Scale (mRS) scores at 1 year were assessed for functional outcomes. Morbidity, mortality, and rebleeding rates were all lower in the MIN group than the craniotomy group $(8.70 \%$ vs. $30.77 \%, 8.70 \%$ vs. $19.23 \%$, and $4.35 \%$ vs. $23.08 \%$, respectively). The 1-year GOSE score was significantly higher in the MIN group than the craniotomy group ( $3.96 \pm 1.55$ vs. $3.10 \pm 1.59, p=0.027$ ). Multivariable logistic regression analysis also revealed that MIN contributed to improved GOSE (estimate: 0.99650, $p=0.0148$ ) and mRS scores (estimate: $-0.72849, p=0.0427$ ) at 1 year. MIN, with low complication rates and improved long-term functional outcome, is feasible and favorable for coagulopathy-related $\mathrm{ICH}$. This promising result should be validated in a large-scale prospective study.

Keywords: intracerebral hemorrhage; coagulopathy; minimally invasive neurosurgery; comparison of surgical outcomes

\section{Introduction}

A significant increase worldwide in patients with coagulopathy-related intracerebral hemorrhage $(\mathrm{ICH})$ has been observed due to the increased use of antithrombotic agents over the last decade for the prevention of cardioembolic events, coronary heart disease, and thrombotic-related disease $[1,2]$. It is estimated that $10-13 \%$ and $27-30 \%$ of ICH patients who underwent surgery were on the treatment of anticoagulant agents and antiplatelet agents, respectively [3,4]. The use of vitamin K antagonists (VKAs) has been associated with $\mathrm{ICH}$ rates of up to $1.8 \%$ per annum, while $\mathrm{ICH}$ rates among novel oral anticoagulant (NOAC)-treated patients are 40-70\% lower than that with warfarin [5]; of particular concern 
is the finding that Asians are four times more likely than Caucasians to develop VKArelated ICH [6]. However, the influence of antithrombotic agents on ICH expansion or neurologic outcomes remains controversial $[3,7,8]$.

In addition to antithrombotic agents, the prevalence of end-stage renal disease (ESRD) among patients receiving hemodialysis in Taiwan is the highest in the world. ICH patients on hemodialysis are exposed to a latent activation of coagulation resulting in an elevated thrombogenic risk, which has been associated with an increased mortality rate ranging from 43.8 to $83 \%$ [9-12].

Rapid reversal of coagulopathy may help to limit ICH expansion and improve clinical outcomes [13]; however, approximately $9.5-33 \%$ of these patients will still require neurosurgical intervention, for whom scant evidence exists as to appropriate surgical management [14-17]. Recent studies have shown promising results with minimally invasive neurosurgery (MIN) in the reduction of mortality and improvement of functional outcomes in ICH patients [18-20]. We therefore retrospectively reviewed our surgical series of coagulopathy-related ICH patients and compared surgical outcomes following traditional craniotomy and MIN.

\section{Materials and Methods}

\subsection{Subjects}

We retrospectively analyzed data from 2013 to 2018 at National Taiwan University Hospital (NTUH), Taipei, Taiwan. Patients with coagulopathy who underwent surgery for ICH with preoperative head computed tomography (CT) and a follow-up head CT within $24 \mathrm{~h}$ after the surgery were included. These patients were not randomly assigned to receive different surgeries. Coagulopathy was defined as (1) using antiplatelet or anticoagulant agents, (2) platelet count $<100 \mathrm{k} / \mu \mathrm{L}$, (3) international normalized ratio $($ INR) $>1.20$, or (4) ESRD on hemodialysis. We excluded patients with vascular lesions (i.e., cerebral aneurysm, arteriovenous malformation, cavernous malformation), cerebellar $\mathrm{ICH}$, traumatic $\mathrm{ICH}$, tumor bleeding, hemorrhagic transformation after ischemic stroke, postoperative ICH, or coagulopathy-related ICH occurring in the intensive care unit (ICU) because these events were often related to systemic diseases, autoimmune dysfunction, acute infection, organ dysfunction, or disseminated intravascular coagulopathy. Patients whose primary treatment was external ventricular drainage (EVD) or decompressive craniectomy were also excluded. This study was approved by the Institutional Review Board of NTUH (approval number: 201611058RINA) and conducted in accordance with the applicable local regulations and the Declaration of Helsinki. Written informed consent was waived due to the use of secondary data.

\subsection{Surgery Selection}

There has been no consensus about the selection of surgical method in coagulopathy patients who require surgical removal of ICH. Even for spontaneous $\mathrm{ICH}$, there are many different methods. In general, neurosurgeons determine the surgical approach (i.e., MIN or craniotomy) according to the location of $\mathrm{ICH}$, type of $\mathrm{ICH}$, and associated comorbidities.

Our consensus for surgical treatment of ICH included (1) a putaminal ICH with a hematoma volume greater than $30 \mathrm{~mL}$, (2) a thalamic ICH with a hematoma volume greater than $20 \mathrm{~mL}$ and IVH with acute hydrocephalus, or (3) a subcortical hemorrhage greater than $30 \mathrm{~mL}$ with significant mass effect (midline shift greater than $5 \mathrm{~mm}$ and effacement of perimesencephalic cistern) [21].

Five major MIN techniques, namely stereotactic thrombolysis, craniopuncture, endoscopic, endoscope-assisted, and endoport-mediated, have been discussed previously [22]; we preferred the endoscope-assisted technique because it has been reported to be associated with better functional outcome and improved cost-effectiveness [23] in the early series, as compared to craniotomy or other MIN techniques. Per our clinical experience, MIN is considered appropriate for deep-seated ICH (i.e., $>1 \mathrm{~cm}$ from the cortical surface), thalamic $\mathrm{ICH}$, putaminal ICH, cerebellar ICH, or ICH with intraventricular hemorrhage (IVH) and 
hydrocephalus. Figure $\mathrm{S} 1$ shows a case with massive ICH for which the accompanying IVH was treated with minimally invasive endoscope-assisted ICH evacuation. For superficial or lobar ICH, craniotomy usually suffices and is straightforward. The selection of surgery was determined solely by the treating surgeon according to the clinical judgment.

All surgeries were performed within $8 \mathrm{~h}$ after the diagnosis of coagulopathy-related ICH to exclude the time to treat factor [18]. In our experience, where more than $84 \%$ of the cases were done within $4 \mathrm{~h}$ after onset, craniectomy was not sufficient to achieve decompression and ICP control [21].

\subsection{MIN Technique}

For most putaminal ICHs, we used the frontal burr hole approach to remove the clot $[23,24]$. In patients with thalamic ICH and IVH, the goal was to relieve the hydrocephalus and elevated ICP while removing the IVH and ICH as much as possible without causing further damage to the brain parenchyma. We therefore used the ipsilateral Kocher's point as our entry point. Since the lateral ventricle was entered during surgery, an EVD was inserted through the operative tract. Bilateral approach with bilateral EVD placement might be considered in extensive IVH cases. Alternatively, using a more lateral trajectory, a septostomy might be done just like in the ventriculoscopic procedure to remove contralateral IVH [25].

Under general anesthesia, a linear skin incision (3-4 cm in length) was made depending on the chosen trajectory. A $1.5 \mathrm{~cm}$ burr hole was created with the dura opened. A $10 \mathrm{~mm}$ corticotomy was then done to insert the transparent plastic sheath $(10 \mathrm{~mm}$ in outer diameter; length $5,7,9$, or $12 \mathrm{~cm}$ depending on the depth of the hematoma) with the stylet. Real-time ultrasound guidance (Aloka UST-5268P-5 neurosurgery burr hole probe, 3.0-7.5 MHz, phased-array sector probe; Aloka, Tokyo, Japan) or navigation system might be helpful before the puncture step. After removing the stylet, the $4 \mathrm{~mm} 0^{\circ}$ endoscope with irrigation system (18 cm in length; Karl Storz, Tuttlingen, Germany) was inserted into the sheath to provide visualization during ICH removal. We used the balanced suction irrigation technique to achieve maximal clot evacuation and inspect bleeders [26]. Local hemostatic agents were very useful in hemostasis during MIN for ICH [27]. If hemostatic agents did not stop the bleeding, the bleeder was identified using the balanced irrigation-suction technique, with constant irrigation and point suction. We usually used commercially available suction coagulation devices (11 Fr, 14 or $19 \mathrm{~cm}$ in length; Kirwan Surgical Products, Marshfield, MA, USA), which performed coagulation and suction simultaneously, to identify and cauterize the bleeder. After ICH removal and hemostasis, an ICP monitor was inserted as needed (according to the guideline, for pre-operative GCS $\leq 8$ or herniation [28]) if the ventricle had not been inserted with an EVD.

\subsection{Endpoints and Variables}

Information on demographics, patient characteristics, medical history, coagulopathy etiology, medication use, preoperative ICH characteristics (i.e., initial Glasgow Coma Scale (GCS), admission ICH score, hematoma volume, presence of IVH), surgical records (i.e., surgical method, surgical duration, blood loss, hematoma clearance rate), and postoperative follow-up data (i.e., rebleeding, length of ICU stay, length of hospital stay, morbidity, GCS, Glasgow Outcome Scale Extended (GOSE), modified Rankin Scale (mRS)) was collected through the electronic medical record system. A higher GOSE score indicates a better functional outcome, while a higher mRS score implies a worse functional outcome. The volume of hematoma was calculated by the $\mathrm{ABC} / 2$ method (A: maximum length in axial cut of $\mathrm{CT}$, B: width perpendicular to A on the same CT cut, and C: the number of slices multiplied by the slice thickness) [29].

The primary endpoints were 1-month rebleeding, morbidity, and mortality rates. The secondary endpoints were hematoma evacuation rate, surgical duration, blood loss, functional outcomes (i.e., GOSE and mRS), and length of ICU/hospital stay. Morbidity included wound dehiscence, surgical site infection, meningitis, ventriculitis, brain abscess, 
and sepsis. We collected postoperative GCS at 1 and 12 months and postoperative GOSE and mRS at 12 months.

\subsection{Statistical Analysis}

The data were first analyzed in a univariate fashion to compare the postoperative outcomes between craniotomy and MIN groups by the independent $t$-test or the Wilcoxon rank-sum test for continuous variables and the chi-square or Fisher's exact test for categorical variables. Multivariate analysis was then performed to explore prognostic factors for postoperative outcomes. All statistical results were declared significant if $p<0.05$. The analysis was performed using Statistical Analysis Software (SAS version 9.4, Cary, NC, USA).

\section{Results}

\subsection{Patient Characteristics}

A total of 362 patients underwent surgery for ICH evacuation at NTUH between 2013 and 2018; 145 of these patients were disqualified due to exclusion criteria. Among the remaining 217 patients, we identified 79 eligible patients who fulfilled the definition of coagulopathy for this study. Four patients were excluded because of loss of long-term follow-up $(n=3)$ and incomplete medical records $(n=1)$, leaving 75 patients who were included in the analysis (Figure 1).

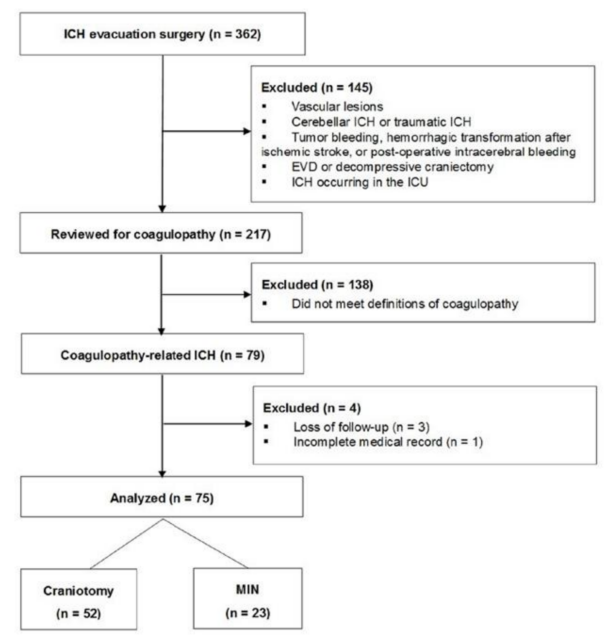

Figure 1. Patient disposition. Abbreviations: EVD, external ventricular drainage; ICH, intracerebral hemorrhage; ICU, intensive care unit; MIN, minimally invasive neurosurgery.

Among enrolled patients, 40 had deep ICH composed of 35 (87.5\%) putaminal ICH and $5(12.5 \%)$ thalamic ICH; the other 35 had lobar ICH, including 12 (34.3\%) frontal lobar ICH, 5 (14.3\%) temporal lobar ICH, 6 (17.1\%) occipital lobar ICH, and 12 (34.3\%) parietal lobar ICH.

Of 75 patients, 52 (33 had lobar ICH and 19 had deep ICH) underwent craniotomy and 23 ( 2 had lobar ICH and 21 had deep ICH) underwent MIN. There were no statistically significant between-group differences in demographics; clinical characteristics regarding coagulopathy such as the use of antithrombotic agents, thrombocytopenia, prolonged prothrombin time, and hemodialysis; or characteristics regarding index ICH such as admission GCS score, admission ICH score, hematoma volume, and the presence of IVH (Table 1). The mean ages in the craniotomy and MIN groups were $58.8 \pm 17.61$ and $60.5 \pm 15.97$ years, respectively. In the craniotomy group, the mean preoperative GCS score was $9.4 \pm 4.06$ and the mean preoperative ICH volume was $62.5 \pm 27.76 \mathrm{~mL}$; corresponding values in the MIN group were $9.4 \pm 3.34$ and $60.7 \pm 24.47 \mathrm{~mL}$, respectively. All 52 patients in the craniotomy group and 22 patients (95.7\%) in the MIN group were administered antiplatelet agents or anticoagulants before the surgery. The antiplatelet agents included 
irreversible cyclooxygenase inhibitors (e.g., aspirin), adenosine diphosphate (ADP) receptor inhibitors (e.g., clopidogrel, ticlopidine), phosphodiesterase inhibitors (e.g., cilostazol), and adenosine reuptake inhibitors (e.g., dipyridamole); anticoagulants included VKAs and NOACs (e.g., dabigatran, rivaroxaban, apixaban).

Table 1. Baseline sociodemographic and clinical characteristics.

\begin{tabular}{lccc}
\hline Variables & $\begin{array}{c}\text { Craniotomy } \\
(\boldsymbol{n}=\mathbf{5 2})\end{array}$ & $\begin{array}{c}\text { MIN } \\
(\boldsymbol{n}=\mathbf{2 3})\end{array}$ & $\boldsymbol{p}$-Value \\
\hline Age (year) & $58.8 \pm 17.61$ & $60.5 \pm 15.97$ & 0.675 \\
Male, $n(\%)$ & $29(55.8)$ & $15(65.2)$ & 0.612 \\
Anticoagulant, $n(\%)$ & $25(48.1)$ & $7(30.4)$ & 0.207 \\
Antiplatelet, $n(\%)$ & $27(51.9)$ & $14(60.9)$ & 0.616 \\
Thrombocytopenia, $n(\%)$ & $9(17.3)$ & $3(13.0)$ & 0.745 \\
Prolonged PT, $n(\%)$ & $21(40.4)$ & $4(17.4)$ & 0.065 \\
Hemodialysis, $n(\%)$ & $3(5.8)$ & $3(13.0)$ & 0.363 \\
GCS & $9.4 \pm 4.06$ & $9.4 \pm 3.34$ & 0.895 \\
IVH, $n(\%)$ & $20(38.5)$ & $14(60.9)$ & 0.084 \\
Hematoma volume $(\mathrm{mL})$ & $62.5 \pm 27.76$ & $60.7 \pm 24.47$ & 0.954 \\
ICH score, $n(\%)$ & $9(17.3)$ & $4(17.4)$ & \\
1 & $21(40.4)$ & $6(26.1)$ & \\
2 & $17(32.7)$ & $8(34.8)$ & \\
3 & $4(7.7)$ & $5(21.7)$ & \\
4 & $1(1.9)$ & $0(0.0)$ & \\
5 & & & \\
\hline
\end{tabular}

Abbreviations: PT, prothrombin time; GCS, Glasgow Coma Scale; IVH, intraventricular hemorrhage; MIN, minimally invasive neurosurgery.

\subsection{Perioperative Records}

Table 2 shows perioperative and postoperative outcomes for the MIN and craniotomy groups. The surgical time in the MIN group (mean, $121.8 \pm 43.92 \mathrm{~min}$ ) was significantly shorter than that in the craniotomy group (mean, $166.4 \pm 63.82 \mathrm{~min})(p=0.003)$. The amount of surgical blood loss in the MIN group (mean, $69.6 \pm 53.81 \mathrm{~mL}$ ) was also significantly smaller than the amount in the craniotomy group (mean, $284.6 \pm 368.56 \mathrm{~mL}$ ) $(p<0.0001)$. There were no between-group differences in the length of ICU or hospital stays or hematoma clearance rates (all $p>0.05$ ).

Table 2. Surgical and postoperative outcomes.

\begin{tabular}{lccc}
\hline Outcome & $\begin{array}{c}\text { Craniotomy } \\
(\boldsymbol{n}=\mathbf{5 2})\end{array}$ & $\begin{array}{c}\text { MIN } \\
(\boldsymbol{n}=\mathbf{2 3})\end{array}$ & $\boldsymbol{p}$-Value \\
\hline Surgical duration $(\mathrm{min})$ & $166.4 \pm 63.82$ & $121.8 \pm 43.92$ & $0.003^{*}$ \\
Surgical blood loss $(\mathrm{mL})$ & $284.6 \pm 368.56$ & $69.6 \pm 53.81$ & $<0.0001^{*}$ \\
ICU stay (days) & $14.8 \pm 9.9$ & $10.7 \pm 5.71$ & 0.162 \\
Hospital stay (days) & $39.6 \pm 31.79$ & $40.0 \pm 12.11$ & 0.642 \\
Hematoma clearance rate $(\%)$ & $77.1 \pm 21.37$ & $80.9 \pm 16.02$ & 0.913 \\
Rebleeding, $n(\%)$ & $12(23.1)$ & $1(4.4)$ & 0.054 \\
Morbidity, $n(\%)$ & $16(30.8)$ & $2(8.7)$ & $0.044^{*}$ \\
Mortality, $n(\%)$ & $10(19.2)$ & $2(8.7)$ & 0.323 \\
GCS on day 30 & $10.9 \pm 4.17$ & $11.3 \pm 3.74$ & 0.770 \\
GCS at 1 year & $10.6 \pm 4.59$ & $11.7 \pm 3.87$ & 0.438 \\
GOSE at 1 year & $3.1 \pm 1.59$ & $4.0 \pm 1.55$ & $0.027 *$ \\
mRS at 1 year & $4.1 \pm 1.36$ & $3.5 \pm 1.34$ & 0.057 \\
\hline
\end{tabular}

Abbreviations: ICU, intensive care unit; MIN, minimally invasive neurosurgery; GCS, Glasgow Coma Scale; GOSE, Glasgow Outcome Scale Extended; mRS, modified Rankin Scale. * Statistical significance.

\subsection{Postoperative Outcomes}

The 1-month morbidity rate in the MIN group (8.7\%) was significantly lower than that in the craniotomy group $(30.8 \%)(p=0.044)$. One-month mortality and rebleeding rates 
were not significantly different between the two groups, but there were more patients who expired or experienced rebleeding within 1 month in the craniotomy group than the MIN group (craniotomy vs. MIN: $19.2 \%$ vs. $8.7 \%$ for mortality, $23.1 \%$ vs. $4.4 \%$ for rebleeding).

As for functional outcomes, the 1-year GOSE score in the MIN group (4.0 \pm 1.55$)$ was significantly higher than that in the craniotomy group $(3.1 \pm 1.59)(p=0.027)$. There were no significant between-group differences in postoperative GCS or mRS, but the mRS score in the MIN group (mean, $3.5 \pm 1.34$ ) was lower than that in the craniotomy group (mean, $4.1 \pm 1.36)$, with borderline significance $(p=0.057)$.

Based on the multivariate linear regression analysis of 1-year functional outcomes (Table 3), MIN was independently associated with the improvement of GOSE (estimate $=0.99650, p=0.0148$ ) and $\mathrm{mRS}$ scores (estimate: $-0.72849, p=0.0427$ ). The preoperative GCS score was found to be an independent predictor of good functional outcomes (1-year GOSE: estimate $=0.15563, p=0.0451$, while anticoagulant therapy was an independent predictor of poor functional outcome (1-year GOSE: estimate $=-1.18483, p=0.0043$; 1-year mRS: estimate $=1.01080, p=0.0059)$.

Table 3. Multivariable linear regression analysis of functional outcomes at 1 year after surgery.

\begin{tabular}{lcccc}
\hline Variables & Estimate & SE & $t$ & $p$-Value \\
\hline Outcome: GOSE at 1 year & & & & \\
\hline Age & -0.00465 & 0.00997 & -0.47 & 0.6423 \\
Male & 0.13788 & 0.33720 & 0.41 & 0.6841 \\
Initial GCS & 0.15563 & 0.07600 & 2.05 & $0.0451^{*}$ \\
Hematoma volume & -0.01230 & 0.00637 & -1.93 & 0.0582 \\
Anticoagulant & -1.18483 & 0.39865 & -2.97 & $0.0043^{*}$ \\
Antiplatelet & -0.31453 & 0.38149 & -0.82 & 0.4130 \\
Hemodialysis & -0.65397 & 0.62699 & -1.04 & 0.3013 \\
Prolonged PT & 0.86207 & 0.37226 & 2.32 & $0.02411^{*}$ \\
Thrombocytopenia & -0.16281 & 0.49824 & -0.33 & 0.7450 \\
IVH & -0.23827 & 0.56119 & -0.42 & 0.6727 \\
Lobar vs. deep ICH & -0.09549 & 0.39400 & -0.24 & 0.8094 \\
MIN vs. craniotomy & 0.99650 & 0.39658 & 2.51 & $0.0148^{*}$ \\
\hline Outcome: mRS at 1 year & & & & \\
Age & 0.00004444 & 0.00883 & 0.01 & 0.9960 \\
Male & -0.28749 & 0.29886 & -0.96 & 0.3401 \\
Initial GCS & -0.12947 & 0.06736 & -1.92 & 0.0595 \\
Hematoma volume & 0.00953 & 0.00564 & 1.69 & 0.0966 \\
Anticoagulant & 1.01080 & 0.35332 & 2.86 & $0.0059^{*}$ \\
Antiplatelet & 0.30615 & 0.33812 & 0.91 & 0.3690 \\
Hemodialysis & 0.27753 & 0.55570 & 0.50 & 0.6194 \\
Prolonged PT & -0.67103 & 0.32994 & -2.03 & $0.0466^{*}$ \\
Thrombocytopenia & 0.02441 & 0.44159 & 0.06 & 0.9561 \\
IVH & 0.27999 & 0.49739 & 0.56 & 0.5757 \\
Lobar vs. deep ICH & 0.09108 & 0.34920 & 0.26 & 0.7951 \\
MIN vs. craniotomy & -0.72849 & 0.35149 & -2.07 & $0.0427^{*}$ \\
\hline Abrian & & & & \\
\hline
\end{tabular}

Abbreviations: PT, prothrombin time; IVH, intraventricular hemorrhage; MIN, minimally invasive neurosurgery; GCS, Glasgow Coma Scale; GOSE, Glasgow Outcome Scale Extended; mRS, modified Rankin Scale; SE, standard error; ICH, intracerebral hemorrhage. * Statistical significance.

The multivariate logistic regression analysis of 1-month clinical events (mortality, rebleeding, and mortality) failed to demonstrate a significant effect for MIN compared with craniotomy (Table 4). Hematoma volume was the only independent predictor of postoperative death, rebleeding, and morbidity (Table 4). 
Table 4. Multivariable logistic regression analysis of clinical events at 1 month after surgery.

\begin{tabular}{|c|c|c|c|c|}
\hline Variables & OR & SE & $95 \% \mathrm{CI}$ & $p$-Value \\
\hline \multicolumn{5}{|c|}{ Outcome: 1-month mortality } \\
\hline Age & 0.950 & 0.0386 & $(0.881,1.025)$ & 0.1835 \\
\hline Male & 0.157 & 1.5195 & $(0.008,3.091)$ & 0.2235 \\
\hline Initial GCS & 0.793 & 0.2640 & $(0.472,1.33)$ & 0.3785 \\
\hline Hematoma volume & 1.047 & 0.0231 & $(1,1.095)$ & $0.0487^{*}$ \\
\hline Anticoagulant & 37.845 & 1.7163 & $(1.309,>999.999)$ & 0.0343 * \\
\hline Antiplatelet & 4.419 & 1.1068 & $(0.505,38.675)$ & 0.1794 \\
\hline Hemodialysis & 29.367 & 2.3717 & $(0.281,>999.999)$ & 0.1541 \\
\hline Prolonged PT & 1.864 & 1.1342 & $(0.202,17.214)$ & 0.5830 \\
\hline Thrombocytopenia & 0.464 & 1.5065 & $(0.024,8.88)$ & 0.6098 \\
\hline IVH & 2.014 & 1.9021 & $(0.048,83.769)$ & 0.7129 \\
\hline Lobar vs. deep ICH & 39.402 & 1.8910 & $(0.968,>999.999)$ & 0.0520 \\
\hline MIN vs. craniotomy & 3.322 & 1.9207 & $(0.077,143.286)$ & 0.5320 \\
\hline \multicolumn{5}{|c|}{ Outcome: 1-month rebleeding } \\
\hline Age & 1.011 & 0.0244 & $(0.964,1.06)$ & 0.6550 \\
\hline Male & 0.492 & 0.8942 & $(0.085,2.839)$ & 0.4277 \\
\hline Initial GCS & 1.046 & 0.1622 & $(0.761,1.438)$ & 0.7795 \\
\hline Hematoma volume & 1.041 & 0.0156 & $(1.009,1.073)$ & $0.0106^{*}$ \\
\hline Anticoagulants & 1.759 & 0.9942 & $(0.251,12.346)$ & 0.5700 \\
\hline Antiplatelet & 1.183 & 0.9136 & $(0.197,7.089)$ & 0.8541 \\
\hline Hemodialysis & $<0.001$ & 209.4 & $(<0.001,>999.999)$ & 0.9601 \\
\hline Prolonged PT & 0.535 & 0.8874 & $(0.094,3.047)$ & 0.4812 \\
\hline Thrombocytopenia & 0.654 & 1.3535 & $(0.046,9.29)$ & 0.7541 \\
\hline IVH & 0.818 & 1.2767 & $(0.067,9.988)$ & 0.8750 \\
\hline Lobar vs. deep ICH & 1.330 & 0.9220 & $(0.218,8.106)$ & 0.7568 \\
\hline MIN vs. craniotomy & 0.094 & 1.3541 & $(0.007,1.337)$ & 0.0809 \\
\hline \multicolumn{5}{|c|}{ Outcome: 1-month morbidity } \\
\hline Age & 0.978 & 0.0197 & $(0.941,1.017)$ & 0.2622 \\
\hline Male & 0.893 & 0.7325 & $(0.212,3.752)$ & 0.8770 \\
\hline Initial GCS & 1.108 & 0.1576 & $(0.814,1.509)$ & 0.5148 \\
\hline Hematoma volume & 1.031 & 0.0143 & $(1.003,1.061)$ & 0.0307 * \\
\hline Anticoagulants & 5.803 & 0.9400 & $(0.92,36.625)$ & 0.0614 \\
\hline Antiplatelet & 1.849 & 0.8598 & $(0.343,9.975)$ & 0.4745 \\
\hline Hemodialysis & 0.764 & 1.4514 & $(0.044,13.139)$ & 0.8529 \\
\hline Prolonged PT & 0.319 & 0.8072 & $(0.066,1.554)$ & 0.1575 \\
\hline Thrombocytopenia & 0.886 & 1.1963 & $(0.085,9.246)$ & 0.9197 \\
\hline IVH & 0.928 & 1.0791 & $(0.112,7.694)$ & 0.9449 \\
\hline Lobar vs. deep ICH & 0.822 & 0.7969 & $(0.172,3.919)$ & 0.8056 \\
\hline MIN vs. craniotomy & 0.199 & 0.9736 & $(0.03,1.344)$ & 0.0977 \\
\hline
\end{tabular}

Abbreviations: PT, prothrombin time; IVH, intraventricular hemorrhage; MIN, minimally invasive neurosurgery; GCS, Glasgow Coma Scale; GOSE, Glasgow Outcome Scale Extended; mRS, modified Rankin Scale; OR, odds ratio; $\mathrm{SE}$, standard error; CI, confidence interval. * Statistical significance.

\subsection{Subgroup Analysis of Anticoagulant and Antiplatelet Agent Cohorts}

Table 5 shows analyses for patients receiving anticoagulants and antiplatelet agents separately. Surgical blood loss in the MIN group was significantly less than that in the craniotomy group for both anticoagulant and antiplatelet agent cohorts. For the anticoagulant cohort, the 1-month morbidity rate in the MIN group (0.0\%) was significantly lower than that in the craniotomy group $(48.0 \%)(p=0.029)$. There were no other significant differences in event rates between the craniotomy and MIN groups in both anticoagulant and antiplatelet agent cohorts; however, more patients in the craniotomy group died, or experienced rebleeding or morbidity within 1 month, compared with the MIN group. 
Table 5. Surgical and postoperative outcomes of anticoagulant and antiplatelet agent cohorts.

\begin{tabular}{|c|c|c|c|c|c|c|}
\hline \multirow[b]{2}{*}{ Outcome } & \multicolumn{3}{|c|}{ Anticoagulants } & \multicolumn{3}{|c|}{ Antiplatelet Agent } \\
\hline & $\begin{array}{l}\text { Craniotomy } \\
\quad(n=25)\end{array}$ & $\begin{array}{l}\text { MIN } \\
(n=7)\end{array}$ & $p$-Value & $\begin{array}{l}\text { Craniotomy } \\
\quad(n=27)\end{array}$ & $\begin{array}{c}\text { MIN } \\
(n=14)\end{array}$ & $p$-Value \\
\hline Surgical duration (min) & $167.7 \pm 59.58$ & $143.1 \pm 42.55$ & 0.362 & $166.3 \pm 63.78$ & $100.7 \pm 32.44$ & $<0.001$ * \\
\hline Surgical blood loss (mL) & $338.0 \pm 443.07$ & $64.3 \pm 24.40$ & $0.006^{*}$ & $255.6 \pm 277.47$ & $60.7 \pm 21.29$ & $<0.001 *$ \\
\hline ICU stay (days) & $16.3 \pm 10.88$ & $8.9 \pm 2.97$ & 0.156 & $16.2 \pm 10.17$ & $10.3 \pm 6.28$ & 0.093 \\
\hline Hospital stay (days) & $34.9 \pm 18.99$ & $27.7 \pm 11.04$ & 0.351 & $45.1 \pm 39.49$ & $29.4 \pm 12.81$ & 0.386 \\
\hline Hematoma clearance rate $(\%)$ & $64.4 \pm 39.32$ & $82.4 \pm 11.21$ & 0.494 & $73.9 \pm 25.50$ & $85.1 \pm 7.30$ & 0.475 \\
\hline Rebleeding, $n(\%)$ & $8(32.0)$ & $0(0.0)$ & 0.150 & $7(25.9 \%)$ & $0(0.0 \%)$ & 0.075 \\
\hline Morbidity, $n(\%)$ & $12(48.0)$ & $0(0.0)$ & $0.029 *$ & $8(29.6 \%)$ & $1(7.1 \%)$ & 0.131 \\
\hline Mortality, $n(\%)$ & $9(36.0)$ & $1(14.3)$ & 0.387 & $5(18.5 \%)$ & $2(14.3 \%)$ & $>0.999$ \\
\hline GCS on day 30 & $9.7 \pm 4.71$ & $10.3 \pm 4.46$ & 0.782 & $10.6 \pm 4.00$ & $10.7 \pm 4.10$ & 0.801 \\
\hline GCS at 1 year & $9.3 \pm 5.30$ & $10.3 \pm 5.02$ & 0.639 & $10.1 \pm 4.53$ & $11.0 \pm 4.47$ & 0.484 \\
\hline GOSE at 1 year & $2.6 \pm 1.55$ & $3.6 \pm 1.90$ & 0.183 & $3.1 \pm 1.67$ & $3.7 \pm 1.73$ & 0.252 \\
\hline mRS at 1 year & $4.5 \pm 1.36$ & $4.0 \pm 1.53$ & 0.412 & $4.1 \pm 1.41$ & $3.7 \pm 1.49$ & 0.354 \\
\hline
\end{tabular}

Abbreviations: ICU, intensive care unit; MIN, minimally invasive neurosurgery; GCS, Glasgow Coma Scale; GOSE, Glasgow Outcome Scale Extended; mRS, modified Rankin Scale. * Statistical significance.

In multivariable linear regression analysis, functional outcomes were better with basal ganglia ICH than with lobar ICH for the anticoagulant cohort, while ICH volume was negatively associated with functional outcomes for the antiplatelet agent cohort (Tables 6 and 7). For both antithrombotic cohorts, multivariable regression analysis failed to reveal significant differences in outcomes according to type of surgery or antithrombotic agents (i.e., VKA or NOAC, clopidogrel, aspirin, or dual antiplatelet).

Table 6. Multivariable linear regression analysis of functional outcomes for anticoagulant and antiplatelet agent cohorts.

\begin{tabular}{|c|c|c|c|c|c|c|c|c|}
\hline \multirow{2}{*}{ Variables } & \multicolumn{4}{|c|}{ Anticoagulants } & \multicolumn{4}{|c|}{ Antiplatelet Agent } \\
\hline & Estimate & SE & $t$ & $p$-Value & Estimate & SE & $t$ & $p$-Value \\
\hline \multicolumn{9}{|l|}{ Outcome: GOSE at 1 year } \\
\hline Age & -0.02307 & 0.02023 & -1.14 & 0.2719 & -0.01130 & 0.01931 & -0.59 & 0.5651 \\
\hline Male & 0.22444 & 0.70776 & 0.32 & 0.7555 & -0.31423 & 0.66402 & -0.47 & 0.6414 \\
\hline Initial GCS & -0.01672 & 0.12771 & -0.13 & 0.8976 & 0.10131 & 0.14283 & 0.71 & 0.4868 \\
\hline Hematoma volume & -0.01394 & 0.01157 & -1.20 & 0.2470 & -0.03190 & 0.01108 & -2.88 & 0.0096 * \\
\hline VKAs vs. NOACs & 0.31520 & 0.82671 & 0.38 & 0.7084 & - & - & - & - \\
\hline Clopidogrel vs. aspirin & - & - & - & - & -1.64591 & 1.09649 & -1.50 & 0.1498 \\
\hline Dual antiplatelet vs. aspirin & - & - & - & - & 1.13290 & 0.92033 & 1.23 & 0.2334 \\
\hline Hemodialysis & -0.78686 & 1.72637 & -0.46 & 0.6551 & -1.53449 & 1.36579 & -1.12 & 0.2752 \\
\hline Prolonged PT & 0.66033 & 0.63047 & 1.05 & 0.3115 & 1.12662 & 0.74057 & 1.52 & 0.1447 \\
\hline Thrombocytopenia & 1.11498 & 0.94553 & 1.18 & 0.2567 & -2.48122 & 1.43867 & -1.72 & 0.1008 \\
\hline IVH & 0.72603 & 0.86924 & 0.84 & 0.4167 & -0.54101 & 1.38124 & -0.39 & 0.6997 \\
\hline Lobar vs. deep ICH & -1.67997 & 0.68970 & -2.44 & $0.0278 *$ & 0.62395 & 0.76093 & 0.82 & 0.4224 \\
\hline MIN vs. craniotomy & 0.91136 & 0.88121 & 1.03 & 0.3174 & 1.23231 & 0.70757 & 1.74 & 0.0977 \\
\hline \multicolumn{9}{|l|}{ Outcome: mRS at 1 year } \\
\hline Age & 0.01383 & 0.01907 & 0.73 & 0.4796 & 0.00436 & 0.01695 & 0.26 & 0.7996 \\
\hline Male & -0.28969 & 0.66721 & -0.43 & 0.6703 & 0.33443 & 0.58296 & 0.57 & 0.5729 \\
\hline Initial GCS & 0.03788 & 0.12039 & 0.31 & 0.7573 & -0.09280 & 0.12540 & -0.74 & 0.4683 \\
\hline Hematoma volume & 0.01357 & 0.01090 & 1.24 & 0.2325 & 0.02616 & 0.00973 & 2.69 & 0.0145 * \\
\hline VKAs vs. NOACs & -0.33501 & 0.77934 & -0.43 & 0.6734 & - & - & - & - \\
\hline Clopidogrel vs. aspirin & - & - & - & - & 1.36025 & 0.96263 & 1.41 & 0.1738 \\
\hline Dual antiplatelet vs. aspirin & - & - & - & - & -0.93597 & 0.80798 & -1.16 & 0.2611 \\
\hline Hemodialysis & 0.91806 & 1.62745 & 0.56 & 0.5810 & 1.10433 & 1.19906 & 0.92 & 0.3686 \\
\hline Prolonged PT & -0.25648 & 0.59434 & -0.43 & 0.6722 & -1.15309 & 0.65016 & -1.77 & 0.0922 \\
\hline Thrombocytopenia & -1.06294 & 0.89136 & -1.19 & 0.2516 & 2.16372 & 1.26305 & 1.71 & 0.1030 \\
\hline IVH & -0.61882 & 0.81944 & -0.76 & 0.4618 & 0.22619 & 1.21263 & 0.19 & 0.8540 \\
\hline Lobar vs. deep ICH & 1.40155 & 0.65019 & 2.16 & $0.0478 *$ & -0.63281 & 0.66804 & -0.95 & 0.3554 \\
\hline MIN vs. craniotomy & -0.08669 & 0.83072 & -0.10 & 0.9183 & -1.07239 & 0.62119 & -1.73 & 0.1005 \\
\hline
\end{tabular}

Abbreviations: PT, prothrombin time; IVH, intraventricular hemorrhage; MIN, minimally invasive neurosurgery; GCS, Glasgow Coma Scale; GOSE, Glasgow Outcome Scale Extended; mRS, modified Rankin Scale; SE, standard error; VKA, vitamin K antagonist; NOAC, novel oral anticoagulant. * Statistical significance. 
Table 7. Multivariable logistic regression analysis of clinical events for anticoagulant and antiplatelet agent cohorts.

\begin{tabular}{|c|c|c|c|c|c|c|c|c|}
\hline \multirow{2}{*}{ Variables } & \multicolumn{4}{|c|}{ Anticoagulants } & \multicolumn{4}{|c|}{ Antiplatelet Agent } \\
\hline & OR & SE & $95 \% \mathrm{CI}$ & $p$-Value & OR & SE & $95 \% \mathrm{CI}$ & $p$-Value \\
\hline \multicolumn{9}{|l|}{ Outcome: 1-month mortality } \\
\hline Age & 1.037 & 0.9644 & $(0.157,6.868)$ & 0.9696 & 0.711 & 3.3646 & $(0.001,519.851)$ & 0.9193 \\
\hline Male & $<0.001$ & 30.0929 & $(<0.001,>999.999)$ & 0.2442 & $>999.999$ & 116.7 & $(<0.001,>999.999)$ & 0.8491 \\
\hline Initial GCS & 0.853 & 7.1359 & $(<0.001,>999.999)$ & 0.9822 & 415.377 & 13.4465 & $(<0.001,>999.999)$ & 0.6539 \\
\hline Hematoma volume & 1.877 & 0.5221 & $(0.674,5.222)$ & 0.2279 & 1.404 & 0.7660 & $(0.313,6.303)$ & 0.6574 \\
\hline VKAs vs. NOACs & 69.362 & 69.7831 & $(<0.001,>999.999)$ & 0.9516 & - & - & - & - \\
\hline Clopidogrel vs. aspirin & - & - & - & - & >999.999 & 180.1 & $(<0.001,>999.999)$ & 0.9227 \\
\hline Dual antiplatelet vs. aspirin & - & - & - & - & $>999.999$ & 48.2435 & $(<0.001,>999.999)$ & 0.4910 \\
\hline Hemodialysis & >999.999 & 140.9 & $(<0.001,>999.999)$ & 0.5534 & $>999.999$ & 81.6034 & $(<0.001,>999.999)$ & 0.6108 \\
\hline Prolonged PT & 5.917 & 13.5302 & $(<0.001,>999.999)$ & 0.8955 & $>999.999$ & 60.4025 & $(<0.001,>999.999)$ & 0.8675 \\
\hline Thrombocytopenia & $<0.001$ & 55.0556 & $(<0.001,>999.999)$ & 0.2236 & $>999.999$ & 77.5521 & $(<0.001,>999.999)$ & 0.6180 \\
\hline IVH & 0.251 & 59.9477 & $(<0.001,>999.999)$ & 0.9816 & $<0.001$ & 105.2 & $(<0.001>999.999)$ & 0.7542 \\
\hline Lobar vs. deep ICH & $>999.999$ & 80.6885 & $(<0.001,>999.999)$ & 0.2323 & $>999.999$ & 87.4267 & $(<0.001,>999.999)$ & 0.9140 \\
\hline MIN vs. craniotomy & $>999.999$ & 71.0683 & $(<0.001,>999.999)$ & 0.8638 & $>999.999$ & 97.5368 & $(<0.001,>999.999)$ & 0.8786 \\
\hline \multicolumn{9}{|l|}{ Outcome: 1-month rebleeding } \\
\hline Age & 3.875 & 2.4295 & $(0.033,453.208)$ & 0.5772 & 1.018 & 1.4983 & $(0.054,19.193)$ & 0.9905 \\
\hline Male & $<0.001$ & 91.3900 & $(<0.001,>999.999)$ & 0.3712 & 0.188 & 52.0048 & $(<0.001,>999.999)$ & 0.9744 \\
\hline Initial GCS & $>999.999$ & 9.1731 & $(<0.001,>999.999)$ & 0.3947 & 9.168 & 9.6752 & $(<0.001,>999.999)$ & 0.8189 \\
\hline Hematoma volume & 3.259 & 1.5559 & $(0.154,68.78)$ & 0.4477 & 1.287 & 0.9458 & $(0.202,8.218)$ & 0.7893 \\
\hline VKAs vs. NOACs & $>999.999$ & 178.1 & $(<0.001,>999.999)$ & 0.6968 & - & - & - & - \\
\hline Clopidogrel vs. aspirin & - & - & - & - & 0.141 & 91.4353 & $(<0.001,>999.999)$ & 0.9829 \\
\hline Dual antiplatelet vs. aspirin & - & - & - & - & 0.005 & 118.4 & $(<0.001,>999.999)$ & 0.9637 \\
\hline Hemodialysis & $>999.999$ & 233.5 & $(<0.001,>999.999)$ & 0.9122 & 817.945 & 123.9 & $(<0.001,>999.999)$ & 0.9568 \\
\hline Prolonged PT & $>999.999$ & 19.2654 & $(<0.001,>999.999)$ & 0.6306 & 0.860 & 123.2 & $(<0.001,>999.999)$ & 0.9990 \\
\hline Thrombocytopenia & $>999.999$ & 127.1 & $(<0.001,>999.999)$ & 0.8106 & 2.663 & 122.1 & $(<0.001,>999.999)$ & 0.9936 \\
\hline IVH & $<0.001$ & 73.0805 & $(<0.001,>999.999)$ & 0.4219 & 0.023 & 96.3720 & $(<0.001,>999.999)$ & 0.9689 \\
\hline Lobar vs. deep ICH & $>999.999$ & 177.1 & $(<0.001,>999.999)$ & 0.8967 & 0.646 & 60.9159 & $(<0.001,>999.999)$ & 0.9943 \\
\hline MIN vs. craniotomy & $>999.999$ & 75.4084 & $(<0.001,>999.999)$ & 0.9026 & $<0.001$ & 62.3455 & $(<0.001,>999.999)$ & 0.8159 \\
\hline \multicolumn{9}{|l|}{ Outcome: 1-month morbidity } \\
\hline Age & 0.293 & 1.3499 & $(0.021,4.132)$ & 0.3634 & 0.617 & 1.5948 & $(0.027,14.058)$ & 0.7622 \\
\hline Male & $<0.001$ & 61.0384 & $(<0.001,>999.999)$ & 0.3363 & $>999.999$ & 78.3938 & $(<0.001,>999.999)$ & 0.8334 \\
\hline Initial GCS & 126.463 & 7.0144 & $(<0.001,>999.999)$ & 0.4902 & 320.953 & 12.7013 & $(<0.001,>999.999)$ & 0.6496 \\
\hline Hematoma volume & 3.332 & 1.2332 & $(0.297,37.359)$ & 0.3291 & 1.573 & 1.3047 & $(0.122,20.288)$ & 0.7285 \\
\hline VKAs vs. NOACs & >999.999 & 124.4 & $(<0.001,>999.999)$ & 0.8893 & - & - & - & - \\
\hline Clopidogrel vs. aspirin & - & - & - & - & $>999.999$ & 110.1 & $(<0.001,>999.999)$ & 0.8439 \\
\hline Dual antiplatelet vs. aspirin & - & - & - & - & $>999.999$ & 103.9 & $(<0.001,>999.999)$ & 0.8643 \\
\hline Hemodialysis & >999.999 & 161.7 & $(<0.001,>999.999)$ & 0.9072 & $>999.999$ & 69.1641 & $(<0.001,>999.999)$ & 0.5552 \\
\hline Prolonged PT & 324.244 & 13.7150 & $(<0.001,>999.999)$ & 0.6734 & 0.002 & 111.7 & $(<0.001,>999.999)$ & 0.9553 \\
\hline Thrombocytopenia & $<0.001$ & 86.9442 & $(<0.001,>999.999)$ & 0.7565 & $>999.999$ & 96.8830 & $(<0.001,>999.999)$ & 0.6754 \\
\hline IVH & $<0.001$ & 65.5522 & $(<0.001,>999.999)$ & 0.5212 & $<0.001$ & 300.3 & $(<0.001,>999.999)$ & 0.9763 \\
\hline Lobar vs. deep ICH & 240.791 & 92.8552 & $(<0.001,>999.999)$ & 0.9529 & $<0.001$ & 96.8247 & $(<0.001,>999.999)$ & 0.6298 \\
\hline MIN vs. craniotomy & 601.171 & 108.4 & $(<0.001,>999.999)$ & 0.9529 & $<0.001$ & 85.5689 & $(<0.001,>999.999)$ & 0.6403 \\
\hline
\end{tabular}

Abbreviations: PT, prothrombin time; IVH, intraventricular hemorrhage; MIN, minimally invasive neurosurgery; GCS, Glasgow Coma Scale; GOSE, Glasgow Outcome Scale Extended; mRS, modified Rankin Scale; OR, odds ratio; SE, standard error; CI, confidence interval; VKA, vitamin K antagonist; NOAC, novel oral anticoagulant.

\section{Discussion}

The present study found that, in patients with coagulopathy-related ICH, MIN is safe and effective compared with craniotomy regarding postoperative outcomes. With its benefits of direct vision via image navigation and reduced surgical blood loss, our results revealed that MIN resulted in lower rates of death, rebleeding, and morbidity, as well as significant improvements in long-term functional outcomes, in comparison with craniotomy in this high-risk cohort.

Recent publications have described promising results with MIN, with decreased mortality and improved functional outcomes compared with traditional craniotomy [4,19,20,30-32]. Two meta-analyses have demonstrated that in selected ICH patients, MIN may be more beneficial than conventional medical treatment or craniotomy [18,33]. A previous study has also reported that endoscopic-assisted MIN was associated with better functional outcomes [23]. Since 2008, our hospital has amassed a dedicated ICH neurosurgical team. To date, we have performed MIN in more than $400 \mathrm{ICH}$ patients and have significantly decreased mortality and improved functional outcomes in these patients $[21,23,27]$. The MIN approach generally evacuates ICH via stereotactic or endoscopic aspiration, with or without thrombolytic usage [28]. We have generally used the endoscopic-assisted 
method because it is faster for decompression and hemostasis can be achieved under direct visualization (as compared to other drainage procedures such as the MISTIE approach).

The pursuit of MIN in coagulopathy-related ICH patients is reasonable, since MIN avoids the inherent collateral damage due to brain retraction during traditional craniotomy, especially in deep-seated lesions, such as a putaminal or thalamic hemorrhage [34,35]. This is particularly important in coagulopathy-related ICH patients, as their vulnerable brain tissue is more prone to intraoperative bleeding or postoperative rebleeding. Such benefits were reflected in our study, which showed a lower rebleeding rate for MIN compared with craniotomy and an association of MIN with significant improvements in long-term functional outcomes. Our study also identified other benefits of MIN, including less blood loss, a shorter operative time, and faster recovery with shorter ICU and hospital stay, compared with craniotomy.

Mortality with anticoagulant-related $\mathrm{ICH}$ has been reported to be as high as $28-33 \%$ [14-17,36]. In the present study, mortality with anticoagulant-related ICH appeared to be lower in the MIN group than in the craniotomy group ( $14 \% \mathrm{vs}$. $36 \%$ ). In the anticoagulant cohort, patients with deep-seated ICH were more likely to have favorable functional outcomes than those with lobar $\mathrm{ICH}$, whereas the type of anticoagulant (NOAC or VKA) did not affect postoperative outcomes. In line with our results, three recent large-scale multicenter observational studies have suggested that baseline ICH volume, hematoma expansion, mortality, and functional outcomes were similar between NOAC- and VKA-related ICH [15,16,37]. Similarly, a cross-sectional survey involving 2245 Japanese patients has revealed no difference in surgical outcomes between VKA- and NOAC-related ICH groups [38]. For the antiplatelet agent cohort, our data suggest that a greater ICH volume may increase the likelihood of poor functional outcomes, yet the postoperative outcomes did not differ between the use of clopidogrel or aspirin. Considering that coagulopathy-related ICH patients may benefit more from MIN than craniotomy in functional outcomes, MIN may be more beneficial for certain coagulopathy patients at higher risk of functional disability, such as anticoagulant-treated patients, patients with poor GCS scores, and antiplatelet-treated patients with ICH expansion.

Besides the additional benefits of MIN in regard to long-term functional outcomes, our study sheds light on minimally invasive surgery for coagulopathy-related ICH by demonstrating that with meticulous perioperative medical management and well-selected surgical options, ICH can be evacuated safely and effectively from patients with coagulopathy. Several other studies have also revealed the benefits of a rapid reversal strategy as the perioperative management for coagulopathy-related $\mathrm{ICH}$ patients undergoing surgery to prevent hemorrhage expansion, limit tissue damage, and facilitate neurosurgical intervention $[13,39,40]$.

Anticoagulant use and low initial GCS scores were found to be independent predictors of poor functional outcomes, whereas hemodialysis, antiplatelet treatment, thrombocytopenia, and the presence of IVH were not correlated with unfavorable functional outcomes or higher risks of postoperative death, rebleeding, and morbidity. Despite the MIN group having more patients with IVH and ICH scores of 4 or above than the craniotomy group, the surgical outcome was not compromised by these factors. Notably, several studies have reported that IVH can be evacuated more easily by MIN than by traditional craniotomy [8,41-43], which may explain why the presence of IVH was not a poor prognostic factor in our study. Moreover, all patients in our study were given prompt reversal of coagulopathy with 24 units of platelet transfusion during the perioperative period to correct the bleeding tendency. We assume that the reversal agents could correct these risk factors if they are used correctly.

This study is limited by its retrospective design, small sample size, and lack of generalizability, as data were collected from a single center. The retrospective design has the potential for informational bias, such as missing data and selection bias based on the surgeon's clinical judgment. Indeed, the surgeon's experience and preference as to the surgical plan were determinant factors for postoperative outcomes. In our study, all patients were 
operated on by the dedicated ICH neurosurgical team, which may have reduced biases from the surgeon's practice. Despite these limitations, this is the first study as far as we know to investigate surgical outcomes of coagulopathy-related $\mathrm{ICH}$ and to determine the optimal surgical option for these patients by comparing MIN and craniotomy. It should be noted that postoperative outcomes following ICH evacuation vary widely across countries. $\mathrm{ICH}-$ related mortality is lower in Asia than in other countries [44]. Evidence also exists as to heterogeneity in efficacy and safety of NOACs and VKAs between Asian and non-Asian populations [45-47]. A multinational, randomized, controlled study that compares MIN and craniotomy for coagulopathy-related ICH is warranted.

\section{Conclusions}

Minimally invasive neurosurgery is safe and effective for coagulopathy-related ICH. It is associated with more favorable surgical and functional outcomes compared with traditional craniotomy. Further prospective, large-scale studies should be initiated to validate this promising result.

Supplementary Materials: The following are available online at https:/ /www.mdpi.com/article/10 $.3390 /$ life11060564/s1, Figure S1. A demonstration of a case with ICH accompanying IVH received minimally-invasive endoscope-assisted ICH evacuation.

Author Contributions: Conceptualization: L.-T.K., D.-M.L. and A.P.-H.H.; methodology: L.-T.K., C.-H.C., W.-M.K., H.-H.T., S.-C.C. and S.-H.Y.; software: Y.-B.L.; validation: Y.-B.L.; formal analysis: Y.-B.L.; resources: L.-T.K., K.-C.W. and A.P.-H.H.; data curation: A.P.-H.H.; writing—original draft preparation: Y.-B.L.; writing-review and editing: C.-H.C. and A.P.-H.H.; visualization: A.P.-H.H.; supervision: D.-M.L.; project administration: A.P.-H.H. All authors have read and agreed to the published version of the manuscript.

Funding: This research received no external funding.

Institutional Review Board Statement: The study was conducted according to the guidelines of the Declaration of Helsinki and approved by the Institutional Review Board of NTUH (approval number: 201611058RINA).

Informed Consent Statement: Patient consent was waived due to secondary-use data. However, the informed consent for the surgery and further publication using nonidentifying information had been obtained from each patient per routine practice.

Data Availability Statement: The data presented in this study are available on request from the corresponding author. The data are not publicly available due to ethical considerations.

Acknowledgments: The authors acknowledge the assistance from Formosa Biomedical Technology Corp. CRO Division in the statistical analysis and editorial support.

Conflicts of Interest: The authors declare no conflict of interest.

\section{References}

1. Dewilde, W.J.; Oirbans, T.; Verheugt, F.W.; Kelder, J.C.; De Smet, B.J.; Herrman, J.-P.; Adriaenssens, T.; Vrolix, M.; Heestermans, A.A.; Vis, M.M.; et al. Use of clopidogrel with or without aspirin in patients taking oral anticoagulant therapy and undergoing percutaneous coronary intervention: An open-label, randomised, controlled trial. Lancet 2013, 381, 1107-1115. [CrossRef]

2. Steinhubl, S.R.; Bhatt, D.L.; Brennan, D.M.; Montalescot, G.; Hankey, G.J.; Eikelboom, J.W.; Berger, P.B.; Topol, E.J. Aspirin to Prevent Cardiovascular Disease: The Association of Aspirin Dose and Clopidogrel With Thrombosis and Bleeding. Ann. Intern. Med. 2009, 150, 379-386. [CrossRef] [PubMed]

3. Liu, Z.-H.; Chen, N.-Y.; Tu, P.-H.; Yip, P.K.; Wang, Y.-C.; Chen, C.-C.; Chuang, C.-C.; Liu, C.-H.; Hsu, P.-W.; Lin, Y.-S. Previous Antithrombotic Therapy, Particularly Anticoagulant, Is Associated with Unfavorable Outcomes in Patients with Primary Spontaneous Intracerebral Hemorrhage Receiving Craniotomy: A Nationwide Population-Based Cohort Study. World Neurosurg. 2019, 128, e59-e73. [CrossRef] [PubMed]

4. Hanley, D.F.; Thompson, R.E.; Rosenblum, M.; Yenokyan, G.; Lane, K.; McBee, N.; Mayo, S.W.; Bistran-Hall, A.J.; Gandhi, D.; Mould, W.A.; et al. Efficacy and safety of minimally invasive surgery with thrombolysis in intracerebral haemorrhage evacuation (MISTIE III): A randomised, controlled, open-label, blinded endpoint phase 3 trial. Lancet 2019, 393, 1021-1032. [CrossRef]

5. Veltkamp, R.; Horstmann, S. Treatment of intracerebral hemorrhage associated with new oral anticoagulant use: The neurologist's view. Clin. Lab. Med. 2014, 34, 587-594. [CrossRef] 
6. Shen, A.Y.-J.; Yao, J.F.; Brar, S.S.; Jorgensen, M.B.; Chen, W. Racial/Ethnic Differences in the Risk of Intracranial Hemorrhage Among Patients with Atrial Fibrillation. J. Am. Coll. Cardiol. 2007, 50, 309-315. [CrossRef]

7. Saloheimo, P.; Ahonen, M.; Juvela, S.; Pyhtinen, J.; Savolainen, E.-R.; Hillbom, M. Regular Aspirin-Use Preceding the Onset of Primary Intracerebral Hemorrhage is an Independent Predictor for Death. Stroke 2006, 37, 129-133. [CrossRef]

8. Stein, M.; Misselwitz, B.; Hamann, G.F.; Kolodziej, M.; Reinges, M.H.; Uhl, E. In-hospital mortality after pre-treatment with antiplatelet agents or oral anticoagulants and hematoma evacuation of intracerebral hematomas. J. Clin. Neurosci. 2016, $26,42-45$. [CrossRef]

9. Iseki, K.; Fukiyama, K. Okawa Dialysis Study (OKIDS) Group Clinical demographics and long-term prognosis after stroke in patients on chronic haemodialysis. Nephrol. Dial. Transplant. 2000, 15, 1808-1813. [CrossRef]

10. Kawamura, M.; Fijimoto, S.; Hisanaga, S.; Yamamoto, Y.; Eto, T. Incidence, outcome, and risk factors of cerebrovascular events in patients undergoing maintenance hemodialysis. Am. J. Kidney Dis. 1998, 31, 991-996. [CrossRef]

11. Murakami, M.; Hamasaki, T.; Kimura, S.; Maruyama, D.; Kakita, K. Clinical Features and Management of Intracranial Hemorrhage in Patients Undergoing Maintenance Dialysis Therapy. Neurol. Med. Chir. 2004, 44, 225-233. [CrossRef]

12. Sakamoto, N.; Ishikawa, E.; Aoki, K.; Uemae, Y.; Komatsu, Y.; Matsumura, A. Clinical Outcomes of Intracerebral Hemorrhage in Hemodialysis Patients. World Neurosurg. 2014, 81, 538-542. [CrossRef]

13. Frontera, J.A.; Lewin, J.J., 3rd; Rabinstein, A.A.; Aisiku, I.P.; Alexandrov, A.W.; Cook, A.M.; del Zoppo, G.J.; Kumar, M.A.; Peerschke, E.I.; Stiefel, M.F.; et al. Guideline for reversal of antithrombotics in intracranial hemorrhage: A statement for healthcare professionals from the neurocritical care society and society of critical care medicine. Neurocrit. Care 2016, 24, 6-46. [CrossRef]

14. Purrucker, J.C.; Haas, K.; Rizos, T.; Khan, S.; Wolf, M.; Hennerici, M.G.; Poli, S.; Kleinschnitz, C.; Steiner, T.; Heuschmann, P.U.; et al. Early Clinical and Radiological Course, Management, and Outcome of Intracerebral Hemorrhage Related to New Oral Anticoagulants. JAMA Neurol. 2016, 73, 169-177. [CrossRef]

15. Gerner, S.T.; Kuramatsu, J.B.; Sembill, J.A.; Sprügel, M.I.; Hagen, M.; Knappe, R.U.; Endres, M.; Haeusler, K.G.; Sobesky, J.; Schurig, J.; et al. Characteristics in Non-Vitamin K Antagonist Oral Anticoagulant-Related Intracerebral Hemorrhage. Stroke 2019, 50, 1392-1402. [CrossRef]

16. Wilson, D.; Seiffge, D.J.; Traenka, C.; Basir, G.; Purrucker, J.C.; Rizos, T.; Sobowale, O.A.; Sallinen, H.; Yeh, S.-J.; Wu, T.Y.; et al. Outcome of intracerebral hemorrhage associated with different oral anticoagulants. Neurology 2017, 88, 1693-1700. [CrossRef]

17. Chen, S.-J.; Yeh, S.-J.; Tang, S.-C.; Lin, S.-Y.; Tsai, L.-K.; Jeng, J.-S. Similar outcomes between vitamin K and non-vitamin K antagonist oral anticoagulants associated intracerebral hemorrhage. J. Formos. Med. Assoc. 2020, 119, 106-112. [CrossRef]

18. Scaggiante, J.; Zhang, X.; Mocco, J.; Kellner, C. Minimally Invasive Surgery for Intracerebral Hemorrhage. Stroke 2018, 49, 2612-2620. [CrossRef]

19. Vespa, P.; Hanley, D.; Betz, J.; Hoffer, A.; Engh, J.; Carter, R.; Nakaji, P.; Ogilvy, C.; Jallo, J.; Selman, W.; et al. Ices (intraoperative stereotactic computed tomography-guided endoscopic surgery) for brain hemorrhage: A multicenter randomized controlled trial. Stroke 2016, 47, 2749-2755. [CrossRef]

20. Gross, B.A.; Jankowitz, B.T.; Friedlander, R.M. Cerebral intraparenchymal hemorrhage: A review. JAMA 2019, 321, 1295-1303. [CrossRef] [PubMed]

21. Kuo, L.-T.; Chen, C.-M.; Li, C.-H.; Tsai, J.-C.; Chiu, H.-C.; Liu, L.-C.; Tu, Y.-K.; Huang, A.P.-H. Early endoscope-assisted hematoma evacuation in patients with supratentorial intracerebral hemorrhage: Case selection, surgical technique, and long-term results. Neurosurg. Focus 2011, 30, E9. [CrossRef] [PubMed]

22. Pan, J.; Chartrain, A.G.; Scaggiante, J.; Spiotta, A.M.; Tang, Z.; Wang, W.; Pradilla, G.; Murayama, Y.; Mori, R.; Mocco, J.; et al. A Compendium of Modern Minimally Invasive Intracerebral Hemorrhage Evacuation Techniques. Oper. Neurosurg. 2019, 18, 710-720. [CrossRef] [PubMed]

23. Cho, D.-Y.; Chen, C.-C.; Chang, C.-S.; Lee, W.-Y.; Tso, M. Endoscopic surgery for spontaneous basal ganglia hemorrhage: Comparing endoscopic surgery, stereotactic aspiration, and craniotomy in noncomatose patients. Surg. Neurol. 2006, 65, 547-555. [CrossRef] [PubMed]

24. Labib, M.A.; Shah, M.; Kassam, A.B.; Young, R.; Zucker, L.; Maioriello, A.; Britz, G.; Agbi, C.; Day, J.D.; Gallia, G.; et al. The safety and feasibility of image-guided brainpath-mediated transsulcul hematoma evacuation: A multicenter study. Neurosurgery 2017, 80, 515-524. [CrossRef]

25. Longatti, P.; Fiorindi, A.; Martinuzzi, A. Neuroendoscopic Aspiration of Hematocephalus Totalis: Technical Note. Oper. Neurosurg. 2005, 57, E409. [CrossRef]

26. Nagasaka, T.; Tsugeno, M.; Ikeda, H.; Okamoto, T.; Takagawa, Y.; Inao, S.; Wakabayashi, T. Balanced irrigation-suction technique with a multifunctional suction cannula and its application for intraoperative hemorrhage in endoscopic evacuation of intracerebral hematomas: Technical note. Neurosurgery 2009, 65, E826-E827. [CrossRef]

27. Luh, H.-T.; Huang, A.P.-H.; Yang, S.-H.; Chen, C.-M.; Cho, D.-Y.; Chen, C.-C.; Kuo, L.-T.; Li, C.-H.; Wang, K.-C.; Tseng, W.-L.; et al. Local hemostatic matrix for endoscope-assisted removal of intracerebral hemorrhage is safe and effective. J. Formos. Med Assoc. 2018, 117, 63-70. [CrossRef]

28. Hemphill, J.C., 3rd; Greenberg, S.M.; Anderson, C.S.; Becker, K.; Bendok, B.R.; Cushman, M.; Fung, G.L.; Goldstein, J.N.; Macdonald, R.L.; Mitchell, P.H.; et al. Guidelines for the management of spontaneous intracerebral hemorrhage: A guideline for healthcare professionals from the american heart association/american stroke association. Stroke 2015, 46, 2032-2060. [CrossRef] 
29. Kothari, R.U.; Brott, T.; Broderick, J.P.; Barsan, W.G.; Sauerbeck, L.R.; Zuccarello, M.; Khoury, J. The ABCs of Measuring Intracerebral Hemorrhage Volumes. Stroke 1996, 27, 1304-1305. [CrossRef]

30. Rennert, R.C.; Signorelli, J.W.; Abraham, P.; Pannell, J.S.; A Khalessi, A. Minimally invasive treatment of intracerebral hemorrhage. Expert Rev. Neurother. 2015, 15, 919-933. [CrossRef]

31. Fiorella, D.; Zuckerman, S.L.; Khan, I.S.; Kumar, N.G.; Mocco, J. Intracerebral Hemorrhage: A Common and Devastating Disease in Need of Better Treatment. World Neurosurg. 2015, 84, 1136-1141. [CrossRef]

32. Hersh, E.H.; Gologorsky, Y.; Chartrain, A.G.; Mocco, J.; Kellner, C.P. Minimally Invasive Surgery for Intracerebral Hemorrhage. Curr. Neurol. Neurosci. Rep. 2018, 18, 34. [CrossRef]

33. Zhou, X.; Chen, J.; Li, Q.; Ren, G.; Yao, G.; Liu, M.; Dong, Q.; Guo, J.; Li, L.; Guo, J.; et al. Minimally invasive surgery for spontaneous supratentorial intracerebral hemorrhage: A meta-analysis of randomized controlled trials. Stroke 2012, 43, 2923-2930. [CrossRef]

34. Adeoye, O.; Woo, D.; Haverbusch, M.; Sekar, P.; Moomaw, C.J.; Broderick, J.; Flaherty, M.L. Surgical management and case-fatality rates of intracerebral hemorrhage in 1988 and 2005. Neurosurgery 2008, 63, 1113-1118. [CrossRef]

35. Zhong, J.; Dujovny, M.; Perlin, A.R.; Perez-Arjona, E.; Park, H.K.; Diaz, F.G. Brain retraction injury. Neurol. Res. 2003, 25, 831-838. [CrossRef]

36. Inohara, T.; Xian, Y.; Liang, L.; Matsouaka, R.A.; Saver, J.L.; Smith, E.E.; Schwamm, L.H.; Reeves, M.J.; Hernandez, A.F.; Bhatt, D.L.; et al. Association of Intracerebral Hemorrhage Among Patients Taking Non-Vitamin K Antagonist vs. Vitamin K Antagonist Oral Anticoagulants With In-Hospital Mortality. JAMA 2018, 319, 463-473. [CrossRef]

37. Boulouis, G.; Morotti, A.; Pasi, M.; Goldstein, J.N.; Gurol, M.E.; Charidimou, A. Outcome of intracerebral haemorrhage related to non-vitamin $\mathrm{K}$ antagonists oral anticoagulants versus vitamin $\mathrm{K}$ antagonists: A comprehensive systematic review and meta-analysis. J. Neurol. Neurosurg. Psychiatry 2018, 89, 263-270. [CrossRef]

38. Kurogi, R.; Nishimura, K.; Nakai, M.; Okada, Y.; Kamitani, S.; Nakagawa, S.; Toyoda, K.; Ogasawara, K.; Kono, K.; Shiokawa, Y.; et al. Comparing intracerebral hemorrhages associated with direct oral anticoagulants or warfarin. Neurol. 2018, 90, e1143-e1149. [CrossRef]

39. Steiner, T.; Weitz, J.I.; Veltkamp, R. Anticoagulant-Associated Intracranial Hemorrhage in the Era of Reversal Agents. Stroke 2017, 48, 1432-1437. [CrossRef]

40. Le Roux, P.; Pollack, C.V.; Milan, M.; Schaefer, A. Race against the clock: Overcoming challenges in the management of anticoagulant-associated intracerebral hemorrhage. J. Neurosurg. 2014, 121, 1-20. [CrossRef]

41. Fiorella, D.; Arthur, A.; Bain, M.; Mocco, J. Minimally invasive surgery for intracerebral and intraventricular hemorrhage: Rationale, review of existing data and emerging technologies. Stroke 2016, 47, 1399-1406. [CrossRef] [PubMed]

42. Li, Y.; Zhang, H.; Wang, X.; She, L.; Yan, Z.; Zhang, N.; Du, R.; Yan, K.; Xu, E.; Pang, L. Neuroendoscopic Surgery versus External Ventricular Drainage Alone or with Intraventricular Fibrinolysis for Intraventricular Hemorrhage Secondary to Spontaneous Supratentorial Hemorrhage: A Systematic Review and Meta-Analysis. PLoS ONE 2013, 8, e80599. [CrossRef] [PubMed]

43. Chen, C.-C.; Liu, C.-L.; Tung, Y.-N.; Lee, H.-C.; Chuang, H.-C.; Lin, S.-Z.; Cho, D.-Y. Endoscopic Surgery for Intraventricular Hemorrhage (IVH) Caused by Thalamic Hemorrhage: Comparisons of Endoscopic Surgery and External Ventricular Drainage (EVD) Surgery. World Neurosurg. 2011, 75, 264-268. [CrossRef] [PubMed]

44. van Asch, C.J.; Luitse, M.J.; Rinkel, G.J.; van der Tweel, I.; Algra, A.; Klijn, C.J. Incidence, case fatality, and functional outcome of intracerebral haemorrhage over time, according to age, sex, and ethnic origin: A systematic review and meta-analysis. Lancet Neurol. 2010, 9, 167-176. [CrossRef]

45. Senoo, K.; Lau, Y.C.; Dzeshka, M.; Lane, D.; Okumura, K.; Lip, G.Y. Efficacy and Safety of Non-Vitamin K Antagonist Oral Anticoagulants vs. Warfarin in Japanese Patients with Atrial Fibrillation. Circ. J. 2015, 79, 339-345. [CrossRef]

46. Cha, M.-J.; Choi, E.-K.; Han, K.-D.; Lee, S.-R.; Lim, W.-H.; Oh, S.; Lip, G.Y. Effectiveness and Safety of Non-Vitamin K Antagonist Oral Anticoagulants in Asian Patients With Atrial Fibrillation. Stroke 2017, 48, 3040-3048. [CrossRef]

47. Wang, K.L.; Lip, G.Y.; Lin, S.J.; Chiang, C.E. Non-vitamin k antagonist oral anticoagulants for stroke prevention in asian patients with nonvalvular atrial fibrillation: Meta-analysis. Stroke 2015, 46, 2555-2561. [CrossRef] 